\title{
Segler im Sonnenwind
}

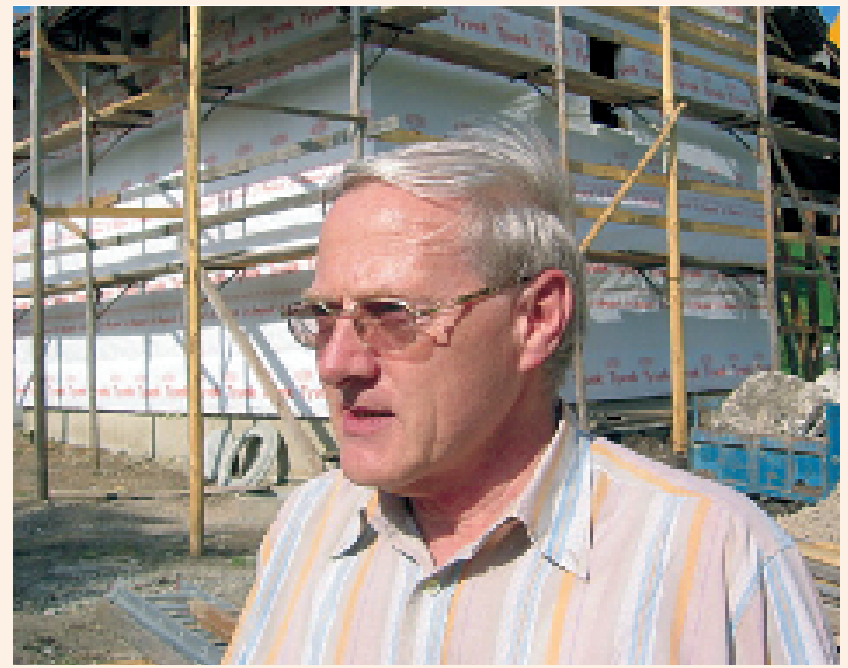

Jede Freizeitminute am Teleskop: Marcel Baer.

Erhard Taverna

Schweizer Astronomie- und Astrophysikexperten sind überall dabei, wo Forscher neue Entdeckungen melden. Genfer beobachten die Milchstrasse und finden in Chile die entfernteste Galaxie. Basler orten einen neuen Sternhaufen, andere leisten Pionierarbeit im Aufspüren extrasolarer Planeten, entdecken Kometen oder entwickeln Geräte für die Robotermission zum Saturnmond Titan. Wie im Spitzensport braucht es auch hier die begabten Amateure, die mit vergleichsweise bescheidenen Mitteln oft Erstaunliches leisten und viel zur Popularität der alten Königsdisziplin Astronomie beitragen.

Der 1943 geborene Marcel Baer, Allgemeinarzt in St. Gallen, gehört zu dieser Spezies engagierter Zeitgenossen, die jede Freizeitminute am Teleskop verbringen. Ein Onkel schulte ihn früh zum Beobachter der Natur und nach einem Schulvortrag des damaligen Präsidenten der Schweizerischen Astronomischen Gesellschaft war auch die Richtung gegeben. Marcel Baer baute sich sein erstes Linsenteleskop aus dem Cosmos-Verlag und hätte gerne Astronomie studiert. Weil das aber als Hungerberuf galt, musste er auf dem Boden bleiben und erst einmal Landvermesser und nach der eidgenössischen Matura Mediziner werden. Er hat das Studium 1979 in Bern mit dem Staatsexamen beendet. Ein bewegliches Spiegelteleskop Marke Maksutow-Cassegrahin, schrittweise ausgebaut mit Refraktor und Spektroskop, begleitete ihn durch alle Weiterbildungs- und Praxisjahre. Wer mit einer H-AlphaAusrüstung, mit Fluoridlinsen und Spezialfiltern im Nanobereich die Sonne erforscht, will sein Werk mit einem Observatorium krönen. Nach einer langen Odyssee schwieriger Standortsuche, verweigerter Zusammenarbeit und abschlägig beantworteter Baugesuche hat die "Amateurund Volkssternwarte Grosser Baer» endlich einen Platz gefunden. Auf dem Areal eines Pflegeheimes entstehen in einer Scheune Arbeits- und Demonstrationsräume, Küche und Werkstatt. Sohn und Schwiegersohn bauen eigenhändig eine mehrstöckige Anlage, in der auf unabhängig fundierten Tragsäulen die optischen Instrumente zu stehen kommen. Im Sommer 2007 wird auf einer Dachgaube die Beobachtungskuppel aus titanoxidbeschichtetem Glasfaserpolyester montiert.

Ein Astronom sitzt nicht jeden Tag am Mittagstisch. Es schwirren die Fragen und Antworten. Berühmte Anlagen werden verglichen, das Hubble Weltraumteleskop, die Riesenspiegel in Chiles Atacama-Wüste und der Vorgänger Mount Palomar. Ein deklassierter Pluto kommen vor, Einschlaglöcher von Shoemaker levi 9 auf dem Jupiter, die Umlaufbahn von Hale-Bopp und technische Details der erfolgreichen Stardustund Cassini-Huygens-Missionen. Auch von gekürzten Budgets ist die Rede, von geschlossenen Planetarien und stillgelegten Observatorien, vom Sparprogramm des Bundes und Personalabbau in europäischen Forschungszentren. Marcel Baer und seine Ehefrau lassen sich ihr Traumziel eine beträchtliche Summe Geld kosten, denn ein Objekt ausserhalb der Bauzone muss zusätzliche Auflagen erfüllen. Dem Mediziner hat es vor allem der Sonnenwind angetan. Er weist darauf hin, dass diese Energieströme nicht nur Polarlichter aufglühen lassen, sondern auch Kraftwerke in Kanada und ICE-Lokomotiven in Deutschland stillgelegt haben. Ihn interessiert 
das Zusammenwirken von Medizin, Biologie und Astrophysik. Für Experimente und Messungen mit Hilfe der Spektralanalyse leistet er sich seine teuren Apparaturen zur Erforschung der Sonne, besonders den Auswirkungen ihrer gewaltigen Energie- und Materieströme auf belebte Organismen.

Marcel Baer ist Mitglied des Vereins Deutscher Sternfreunde und korrespondiert mit deren Fachgruppe für Spektroskopie. Sein Projekt beinhaltet mehrere Schwerpunkte: öffentliche Auftritte mit der Ortsschule und dem entstehenden Reka-Feriendorf, Videokameraaufnahmen von Himmelskörpern und Fotografien der Sonnenprotuberanzen. Seine grosse Hoffnung sind die «ultrafeinen» oder «morphogenetischen» Ener- giefelder, die er mit seiner Spektralanalyse untersuchen möchte. Wer sich so weit ins All hinauswagt, bewegt sich immer schon in Grenzgebieten. Die Astronomie ist aus der Astrologie hervorgegangen, wie die Chemie aus der Alchemie. Vielleicht finden Wissenschaft und Esoterik im «Grossen Baer» einen gemeinsamen Nenner, vielleicht ist es wieder einmal ein Aussenseiter, der wie Schliemann das sagenhafte Troja entdeckt. Ich habe ihn nicht gefragt, ob er «Kraftorte der Schweiz» von Blanche Merz gelesen hat. Am Gegenhang seiner Sternwarte liegt eine Superzone des Erdmeridians, mit 75000 Boviseinheiten das zweitgrösste Energiefeld der Schweiz. Die Chancen für Marcel Baer stehen nicht schlecht. 